\title{
On the Mechanism of Low-Temperature Termolecular Atomic Recombination
}

\author{
Russell T. Pack \\ rt7mhpack@msn.com \\ Richard L. Snow \\ Wesley D. Smith
}

Follow this and additional works at: https://scholarsarchive.byu.edu/facpub

Part of the Biochemistry Commons, and the Chemistry Commons

\section{Original Publication Citation}

Pack, Russell T., Richard L. Snow, and Wesley D. Smith."On the Mechanism of Low-Temperature

Termolecular Atomic Recombination." The Journal of Chemical Physics 56 (1972): 926-932.

\section{BYU ScholarsArchive Citation}

Pack, Russell T.; Snow, Richard L.; and Smith, Wesley D., "On the Mechanism of Low-Temperature Termolecular Atomic Recombination" (1972). Faculty Publications. 792.

https://scholarsarchive.byu.edu/facpub/792

This Peer-Reviewed Article is brought to you for free and open access by BYU ScholarsArchive. It has been accepted for inclusion in Faculty Publications by an authorized administrator of BYU ScholarsArchive. For more information, please contact ellen_amatangelo@byu.edu. 


\title{
On the Mechanism of Low-Temperature Termolecular Atomic Recombination*
}

\author{
Russell T Pack, Richard L. Snow, and Wesley D. Smitu \\ Department of Chemistry, Brigham Young University, Prmo, Utah 84601
}

(Received 9 September 1971)

\begin{abstract}
A simple theory of gas-phase termolecular atomic recombination kinetics is presented which treats the bound-complex (BC) mechanism in a fashion which parallels the Roberts, Bernstein, and Curtiss resonance theory of the energy-transfer (ET) mechanism. The ET rate constant has a low temperature maximum, but the $\mathrm{BC}$ rate constant does not. The contributions of the two mechanisms to the low-temperature recombination of hydrogen atoms, with $\mathrm{He}, \mathrm{Ar}$, and $\mathrm{H}_{2}$ as third bodies, are estimated and compared. The presence (absence) of a low-temperature maximum in the observable total rate constant is determined by absence (presence) of bound vibrational levels of the hydrogen-third body van der Waals dimer. Hence, experimental studies of these reactions could yield valuable information about the interatomic potentials.
\end{abstract}

\section{INTRODUCTION}

It has long been known ${ }^{1}$ that the gas-phase termolecular recombination of $\mathrm{X}$ atoms in the presence of thirdbody atoms or molecules $\mathrm{M}$,

$$
\mathrm{X}+\mathrm{X}+\mathrm{M} \rightarrow \mathrm{X}_{2}+\mathrm{M},
$$

can occur by two distinct mechanisms involving successive two-body collisions. These are the energytransfer (ET) mechanism, summarized by

$$
\begin{gathered}
\mathrm{X}+\mathrm{X} \rightleftharpoons \mathrm{X}_{2}{ }^{*}, \\
\mathrm{X}_{2}{ }^{*}+\mathrm{M} \rightarrow \mathrm{X}_{2}+\mathrm{M},
\end{gathered}
$$

and the bound-complex (BC) or radical-molecule complex mechanism, summarized by

$$
\begin{aligned}
\mathrm{X}+\mathrm{M} & \rightleftharpoons \mathrm{MX}, \\
\mathrm{MX}+\mathrm{X} & \rightarrow \mathrm{X}_{2}+\mathrm{M} .
\end{aligned}
$$

A number of theoretical calculations ${ }^{2-6}$ have been made to compare the importance of the two mechanisms, and it has generally been agreed ${ }^{2-4}$ that the $\mathrm{BC}$ mechanism is dominant if the intermolecular potential between $\mathrm{X}$ and $\mathrm{M}$ is strongly attractive. However, when $M$ is a noble-gas atom, there has been disagreement about whether the $\mathrm{BC}^{5}$ or the $\mathrm{ET}^{6}$ mechanism is the more important. Furthermore, some authors have simply assumed that either the $\mathrm{BC}^{7}$ or the $\mathrm{ET}^{8-11}$ mechanism was the only important one. One reason for this confusion is that the theories used for the two mechanisms have often been nonequivalent and have given unreasonable results for one or the other mechanism. In this paper we attempt to keep the methods used for the two mechanisms as alike as possible.

The most accurate and detailed calculations of the contribution of the ET mechanism are the resonance theory calculations of Roberts, Bernstein, and Curtiss ${ }^{10}$ (RBC) on the recombination of $\mathrm{H}$ atoms in $\mathrm{H}_{2}, \mathrm{He}$, and Ar. A distinguishing feature of the $\mathrm{RBC}$ results is a predicted maximum in the rate constant at about $100^{\circ} \mathrm{K}$. However, Ham, Trainor, and Kaufman ${ }^{12}$ studied the low-temperature recombination of $\mathrm{H}$ in $\mathrm{H}_{2}$ experimentally to check this feature and found no maximum. Calculations by Shui and Appleton ${ }^{13}$ using
Keck' ${ }^{14,15}$ classical phase-space theory (which includes contributions from both mechanisms) also show no maximum and agree well with the experimental data. Hence, one is led to suspect that the loss of the maximum is due to contributions by the $\mathrm{BC}$ mechanism.

Although simple phase-space theories such as those of Keck ${ }^{13-15}$ are about as accurate as justified by presently available intermolecular potentials and experimental rate constants, they include both the $\mathrm{BC}$ and ET mechanisms automatically and cannot resolve the two contributions. Also, it is not clear whether the lack of a maximum in the phase-space theory rate constant is due to the $\mathrm{BC}$ mechanism or to the neglect of all quantum effects. Thus, in order to understand the presence or absence of a maximum in the observable rate constant one needs to know the separate contributions of the two mechanisms.

In the following section of this paper we first summarize and simplify the $\mathrm{RBC}$ theory in a way which still gives reasonable estimates of the ET contribution to the recombination rate. This allows development (in Sec. III) of a theory for the BC mechanism which parallels our treatment of the ET mechanism. In Sec. IV we review a simple method ${ }^{16}$ for determining the bound and quasibound rotation-vibration states of a diatomic molecule. The results of our calculations are presented in Sec. V and compared with other available results. And finally, Sec. VI contains a discussion of those results.

\section{ENERGY-TRANSFER MECHANISM}

We now summariz e the theory of Roberts, Bernstein and Curtiss ${ }^{10}(\mathrm{RBC})$ for the contribution of the energytransfer (ET) mechanism to the recombination rate. (Justification of the assumptions involved is found in RBC's work.)

For the recombination of $\mathrm{X}$ atoms in the presence of a third body, M, the detailed ET mechanism is

$$
\begin{array}{cc}
\mathrm{X}+\mathrm{X} \underset{k d^{i}}{\stackrel{k_{f}^{i}}{\rightleftharpoons}} \mathrm{X}_{2}^{i}, & \left(E_{i} \geq 0\right), \\
\mathrm{X}_{2}{ }^{i}+\mathrm{M} \stackrel{k_{f}^{i n}}{\longrightarrow} \mathrm{X}_{2}{ }^{n}+\mathrm{M}, & \left(E_{n}<0\right) .
\end{array}
$$


Here the complexes, $i$, are quasibound rotation-vibration states (orbiting resonances) of $\mathrm{X}_{2}$ made possible by the rotational barrier in the effective potential, and the states, $n$, are true bound states with lower energy than the separated atoms.

In order to obtain the simple third-order kinetics observed experimentally for reactions of this type, it is necessary to assume that all the quasibound states that contribute appreciably to the recombination rate are in equilibrium with the reactants. Then, the over-all rate of formation of $\mathrm{X}_{2}$ by the ET mechanism is

$$
d n_{\mathrm{X}_{2}} / d t=k_{r}{ }^{\mathrm{ET}} n_{\mathrm{X}^{2}} n_{\mathrm{M}},
$$

where $n$ is the number density of the subscripted molecule, and the ET recombination rate constant is given by

$$
k_{r}^{\mathrm{ET}}=\sum_{i} \sum_{n} k_{f}^{i n} K_{\mathrm{m} n}^{i}
$$

Here $K_{\mathrm{eq}}{ }^{i}=k_{f}{ }^{i} / k_{d}{ }^{i}$ is the equilibrium constant for the formation of $\mathrm{X}_{2}$ molecules in the $i$ th quasibound state. It can be calculated from equilibrium statistical mechanics using

$$
\begin{aligned}
K_{\mathrm{eq}}{ }^{i}=\left(g_{i} / g_{\mathrm{X}}{ }^{2}\right) & \left(2 J_{i}+1\right) \\
& \times \exp \left(-E_{i} / k T\right)\left(2 \pi \hbar^{2} / \mu_{\mathrm{X}_{2}} k T\right)^{3 / 2},
\end{aligned}
$$

where $g_{i}$ is the electronic and nuclear degeneracy of $\mathrm{X}_{2}{ }^{i}, g_{\mathrm{X}}$ the degeneracy of $\mathrm{X}, J_{i}$ the rotational quantum number, $E_{i}$ the energy of the $i$ th state relative to the separated atoms, and $\mu_{\mathrm{X}_{2}}=\frac{1}{2} m_{\mathrm{X}}$ the reduced mass of $\mathrm{X}_{2}$.

The bimolecular specific rate constants, $k_{f}{ }^{\text {in }}$ in (2.4) are related to the inelastic scattering cross sections for the transition from state $i$ to state $n$ by the equation

$$
k_{f}^{i n}=\left(8 k T / \pi \mu_{\mathrm{X}_{2}-\mathrm{M}}\right)^{1 / 2} \overline{\boldsymbol{\sigma}}_{i n},
$$

where $\mu_{\mathrm{X}_{2}-\mathrm{M}}=m_{\mathrm{M}} m_{\mathrm{X}_{2}} /\left(m_{\mathrm{M}}+m_{\mathrm{X}_{2}}\right)$ is the appropriate reduced mass, and $\bar{\sigma}_{i n}$ is the Boltzmann average of the cross section $\sigma_{\text {in }}(E)$,

$$
\bar{\sigma}_{i n}=(k T)^{-2} \int_{0}^{\infty} \sigma_{\text {in }}(E) E e^{-E / k T} d E,
$$

$E$ being the relative energy of the $\mathrm{X}_{2}{ }^{i}-\mathbf{M}$ collision. Letting $\bar{\sigma}_{i}$ be the total inelastic cross section for a transition from state $i$ to any bound state,

$$
\bar{\sigma}_{i}=\sum_{n} \bar{\sigma}_{i n},
$$

and using (2.6) in (2.4), one obtains

$$
k_{r} \mathrm{ET}=\left(8 k T / \pi \mu_{\mathrm{X}_{2}-\mathrm{M}}\right)^{1 / 2} \sum_{i} K_{\mathrm{eq}}{ }^{i} \bar{\sigma}_{i} .
$$

$\mathrm{RBC}$ calculated $k_{\mathrm{r}}{ }_{\mathrm{ET}}$ using the quasibound $\mathrm{H}_{2}$ spectrum of Waech and Bernstein ${ }^{17}$ to get $K_{\mathrm{eq}}{ }^{i}$ and a restricted distorted-wave approximation with dumbell model potentials to get $\bar{\sigma}_{i}$. From the results they noted that $\sigma_{i n}(E)$ varied slowly with $E$, so that, to a good approximation, (2.7) becomes

$$
\bar{\sigma}_{i n}=\sigma_{i n}(k T) \text {. }
$$

In addition, $\mathrm{RBC}$ found that a useful upper bound to $\bar{\sigma}_{i}$ was given by the simple hard-sphere gas-kinetic cross section,

$$
\sigma_{i}^{\mathrm{GK}}(k T)=\pi\left(r_{\mathrm{MX}}+\frac{1}{2}\left\langle r^{2}\right\rangle_{i}{ }^{1 / 2}\right)^{2},
$$

where $r_{M X}$ is the classical turning point (obtained from a model potential) for an $\mathbf{M}-\mathbf{X}$ collision with energy $k T$, and $\left\langle r^{2}\right\rangle_{i}{ }^{1 / 2}$ is the root-mean-square internuclear distance of complex $\mathrm{X}_{2}{ }^{i}$.

Let us now modify these last two equations to obtain a simple but reasonable estimate for $\bar{\sigma}_{i}$. If we let $\bar{\sigma}_{i n}$ be given by

$$
\bar{\sigma}_{i n}=\sigma_{i n}(k T)=P_{i n} \sigma_{i}^{\mathrm{GK}}(k T),
$$

where $P_{i n}$ is the probability of a transition from state $i$ to state $n$, then from (2.8) we have

$$
\vec{\sigma}_{i}=P_{i} \sigma_{i}^{\mathrm{GK}}(k T),
$$

where $P_{i}$ is the probability of a transition to any bound state,

$$
P_{i}=\sum_{n} P_{i n} \leq 1 .
$$

In $\mathrm{X}_{2}{ }^{i}-\mathrm{M}$ collisions, we expect that the sum of the probabilities of elastic collisions, of transitions to unbound states, and of transitions to other quasibound states is at least as large as $P_{i}$; hence, we require that $P_{i} \leq \frac{1}{2}$. In fact, we take

$$
P_{i}=\frac{1}{2}\left(\delta_{i} / \sinh \delta_{i}\right)^{2} .
$$

Here $P_{i}$ is further decreased by a factor which arises from the theory of inelastic collisions between systems interacting by means of a repulsive exponential potential, ${ }^{10,18,19}$

$$
V(\boldsymbol{r})=\epsilon \exp (-\boldsymbol{r} / a)
$$

In terms of the steepness parameter, $a$, of this potential, $\delta_{i}$ is given by ${ }^{10,18,19}$

$\delta_{i}(k T)=\pi\left(2 \mu_{\mathrm{X}_{2}-\mathrm{M}} a^{2} / \hbar^{2}\right)^{1 / 2}\left[\left(k T+E_{i}\right)^{1 / 2}-(k T)^{1 / 2}\right]$.

The factor involving $\delta_{i}$ in (2.15) ensures that $P_{i}$ becomes small if $E_{i}$ is large; in other words, the probability of a transition from state $i$ to the bound region is small if $i$ lies far from that region.

With these approximations, (2.9) becomes

$k_{r}^{\mathrm{ET}}=\left(8 k T / \pi \mu_{\mathrm{X}_{2}-\mathrm{M}}\right)^{1 / 2} \sum_{i} P_{i}(k T) \sigma_{i}^{\mathrm{GK}}(k T) K_{\mathrm{eq}}{ }^{\mathrm{G}}$.

In order to actually calculate $k_{r}{ }^{\mathrm{ET}}$, one needs to know the energies, $E_{i}$, and the internuclear distances, $\left\langle r^{2}\right\rangle_{i}{ }^{1 / 2}$, of those quasibound states that contribute appreciably to the rate; that is, those states which form and dissociate rapidly enough to maintain equilibrium with the reactant atoms yet have lifetimes much longer than those of nonresonant $X-X$ collisions. (Specific criteria have been given by $R B C$.) Then, one needs an $M-X$ potential of the form (2.16) to calculate $\delta_{i}$ and $\boldsymbol{r}_{\mathrm{Mx}}$. 
Substitution into (2.18) and summation gives the desired estimate of $k_{r} E \mathrm{~T}$.

\section{BOUND-COMPLEX MECHANISM}

We now consider the bound-complex (BC) mechanism and develop a treatment of it which parallels the $\mathrm{RBC}$ theory for the ET mechanism. In detail the BC mechanism is

$$
\begin{aligned}
& \mathrm{X}+\mathrm{M} \underset{k_{2}{ }^{i}}{\stackrel{k_{1}{ }^{i}}{\rightleftharpoons}} \mathrm{MX}^{i}, \quad\left(E_{i} \geq 0\right), \\
& \mathrm{MX}^{i}+\mathrm{M} \underset{k_{4}^{i m}}{\stackrel{k_{3}^{i m}}{\rightleftharpoons}} \mathrm{MX}^{m}+\mathrm{M}, \\
& \mathrm{X}+\mathrm{M}_{2} \underset{k_{6^{m}}}{\stackrel{k_{5}{ }^{m}}{\longrightarrow}} \mathrm{MX}^{m}+\mathrm{M},
\end{aligned}
$$

and

$$
\mathrm{X}+\mathrm{MX}^{m} \stackrel{k_{f}^{m n}}{\longrightarrow} \mathrm{X}_{2}{ }^{n}+\mathrm{M}, \quad\left(E_{n}<0\right) .
$$

Equations (3.1) describe some possible processes for the formation and equilibration of MX dimers. Here $m$ may be a bound $\left(E_{m}<0\right)$ or a quasibound $\left(E_{m} \geq 0\right)$ rotation-vibration state of $\mathrm{MX}$. In order to obtain third-order kinetics, we assume that all those dimer states $\mathrm{MX}^{m}$ that contribute appreciably to (3.2) are in equilibrium with $\mathrm{X}$ and $\mathrm{M}$. Then the rate of formation of bound $\mathbf{X}_{2}$ molecules via this mechanism is

where

$$
d n_{\mathrm{X}_{2}} / d t=k_{r}{ }^{\mathrm{BC}} n_{\mathrm{X}^{2}} n_{\mathrm{M}},
$$

$$
k_{r}{ }^{\mathrm{BC}}=\sum_{m} \sum_{n} k_{f}^{m n} K_{\mathrm{eq}}{ }^{m} .
$$

Although this expression has the same appearance as (2.4), $m$ now runs over bound states as well as quasibound, and $k_{f}{ }^{m n}$ is the specific rate constant for the exchange reaction (3.2) rather than the inelastic collision (2.2).

If an MX interatomic potential is known from which the properties of the rotation-vibration states of $\mathrm{XM}^{m}$ can be calculated, then $K_{\text {eq }}{ }^{m}$ can be obtained from equilibrium statistical mechanics,

$$
\begin{aligned}
K_{\mathrm{eq}}{ }^{m}=\left(g_{m} / g_{\mathrm{X}} g_{\mathrm{M}}\right)\left(2 J_{m}+1\right) & \\
& \times \exp \left(-E_{m} / k T\right)\left(2 \pi \hbar^{2} / \mu_{\mathrm{MX}} k T\right)^{3 / 2},
\end{aligned}
$$

where $\mu_{\mathrm{MX}}=m_{\mathrm{X}} m_{\mathrm{M}} /\left(m_{\mathrm{X}}+m_{\mathrm{M}}\right)$, and the other terms are as defined at $(2.6)$.

Before discussing the determination of $k_{f}{ }^{m n}$ in (3.4), let us consider just which states $m$ contribute appreciably. If $\mathrm{M}$ were chemically reactive, so that $\mathrm{MX}$ had many bound levels, one might expect the lowest levels of MX to be populated so slowly that they would contribute negligibly to the recombination rate. However, here we restrict $M$ to be a chemically inert molecule or noble-gas atom, so that the few existing van der Waals dimers $\mathrm{MX}^{m}$ are all weakly bound. These should all be readily populated, and we expect MX to be a "relaxed" complex (one in which all the states are in equilibrium with $\mathrm{M}$ and $\mathrm{X}$ ). Shui, Appleton, and Keck $^{14}$ give the condition for a relaxed complex formed via (3.1a) and (3.1b) as

$$
\exp \left(-\epsilon_{\mathbf{M X}} / k T\right)>n_{\mathbf{X}} / n_{\mathbf{M}},
$$

where $\epsilon_{M X}$ is the depth of the MX potential well. For the specific problems considered in this paper, this condition holds for all $T>20^{\circ} \mathrm{K}$. Furthermore, Reaction (3.1c) also produces $\mathrm{MX}$ dimers from $\mathrm{M}_{2}$ dimers (provided they exist). This reaction is expected to have zero $^{20}$ activation energy thus keeping the complexes relaxed at even lower temperatures. Therefore, a relaxed complex was assumed at all temperatures of interest.

The specific bimolecular rate constants $k_{f}^{m n}$ are related to the cross sections for the reactive collision (3.2) by equations similar to (2.6) and (2.7). To calculate $k_{r}{ }^{B C}$ accurately one must calculate accurate reactive cross sections. At present, the theory of reactive scattering is not well developed, and the calculation of accurate reactive cross sections requires heavy computations hardly justified by the crude intermolecular potential surfaces available. Hence, we seek a simpler way to estimate the needed cross sections. Reaction (3.2) has a negligible activation energy, ${ }^{20}$ so that $\sigma_{m n}(E)$ is expected to be of gas-kinetic order and to vary slowly with $E$. Then, Eq. (2.10) holds and (3.4) reduces to

$$
k_{r}{ }^{\mathrm{BC}}=\left(8 k T / \pi_{\mu_{\mathrm{MX}}-\mathrm{X}}\right)^{1 / 2} \sum_{m} K_{\mathrm{eq}}{ }^{m} \bar{\sigma}_{m},
$$

where $\mu_{\mathrm{MX}-\mathrm{X}}=m_{\mathrm{MX}} m_{\mathrm{X}} /\left(m_{\mathrm{MX}}+m_{\mathrm{X}}\right)$.

Once again, we take

$$
\bar{\sigma}_{m}=P_{m}(k T) \sigma_{m}{ }^{\mathrm{GK}}(k T),
$$

where $P_{m}=\sum_{n} P_{m n}$ is the total probability of a reaction producing a bound $\mathrm{X}_{2}$ molecule. For $\sigma_{m}{ }^{\mathrm{GK}}$ we use the expression

$$
\sigma_{m}{ }^{\mathrm{GK}}=\pi\left[\left(r_{\mathrm{XX}} r_{\mathrm{MX}}\right)^{1 / 2}+\frac{1}{2}\left\langle r^{2}\right\rangle_{m}^{1 / 2}\right]^{2},
$$

where $r_{\mathrm{XX}}$ is the classical turning point for a collision of energy $k T$ between two $\mathrm{X}$ atoms. For the reaction probability, $P_{m}$, in the case that $E_{m}<0$, we take

$$
P_{m}=\frac{1}{3} G,
$$

where $\frac{1}{3}$ is chosen as a reasonable steric factor, and $G$ is the probability that the $\mathrm{MX}-\mathrm{X}$ collision occurs on a potential-energy surface that can lead to bound $\mathrm{X}_{2}$ molecules. For $E_{m} \geq 0$, we use ${ }^{21}$

$$
P_{m}=\frac{1}{3} G\left(\delta_{m} / \sinh \delta_{m}\right)^{2} .
$$

Here, $P_{m}$ is smaller than for $E_{m}<0$ because the successful reactive collisions must be vibrationally nonadiabatic and because the probability that the MX-X collision produces dissociation of MX is greater. In (3.11) we put ${ }^{21}$

$\delta_{m}(k T)=\pi\left(2 \mu_{\mathrm{MX}-\mathrm{x}} b^{2} / \hbar^{2}\right)^{1 / 2}\left[\left(k T+E_{m}\right)^{1 / 2}-(k T)^{1 / 2}\right]$, 
where $b$ is the steepness parameter of the repulsive part of the MX-X potential.

With these approximations, the $\mathrm{BC}$ rate constant is

$$
k_{\tau}^{\mathrm{BC}}=\left(8 k T / \pi \mu_{\mathrm{MX}-\mathrm{X}}\right)^{1 / 2} \sum_{m} P_{m}(k T) \sigma_{m}{ }^{\mathrm{GK}}(k T) K_{\mathrm{eq}}{ }^{m} \text {. }
$$

Using available interatomic potentials the rotationvibration states of $\mathrm{MX}$ can be calculated as described in the next section, and with them $k_{r}{ }^{\mathrm{BC}}$ is easily determined.

\section{ROTATION-VIBRATION STATES OF MX}

To determine the rotation-vibration states of $\mathrm{MX}$, we apply Connor's ${ }^{16}$ quasiclassical WKB methods. We now outline the procedures followed.

For bound states $\left(E_{m}<0\right)$ of $\mathrm{MX}$, the energy levels were calculated by Gaussian integration of the potential-well phase integral (see Fig. 1),

$$
\phi=\pi^{-1}\left(\frac{2 \mu_{\mathrm{MX}}}{\hbar^{2}}\right)^{1 / 2} \int_{r_{1}}^{r_{2}}\left(E-V_{J}\right)^{1 / 2} d r
$$

where $V_{J}$ is the effective potential

$$
V_{J}=V(r)+\hbar^{2} J(J+1) / 2 \mu_{\mathrm{MX}} r^{2}
$$

The energies, $E_{m}<0$, were determined by the usual Bohr-Sommerfeld quantization condition

$$
\phi=\left(\nu_{m}+\frac{1}{2}\right), \quad \nu_{m}=0,1,2, \cdots
$$

The root-mean-square internuclear distance was obtained from the WKB expectation value formula

$$
\begin{aligned}
\left\langle r^{2}\right\rangle_{m}{ }^{1 / 2}=\left[\int_{r 1}^{r 2}\left(E_{m}-V_{J}\right)^{-1 / 2} r^{2} d r\right]^{1 / 2} & \\
& \times\left[\int_{r_{1}}^{r 2}\left(E_{m}-V_{J}\right)^{-1 / 2} d r\right]^{-1 / 2} .
\end{aligned}
$$

The energies of the quasibound states $\left(E_{m} \geq 0\right)$ were determined using Connor's correction ${ }^{16}$

$$
\phi+(2 \pi)^{-1} \chi=\left(\nu_{m}+\frac{1}{2}\right),
$$

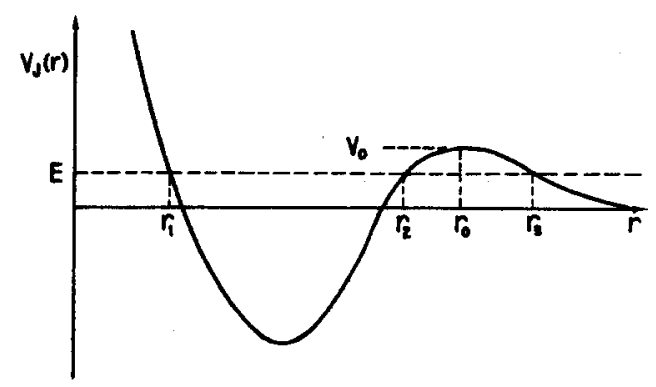

Fig. 1. Sketch of an effective interatomic potential, $V_{J}(r)$, for angular momentum $J \neq 0 . r_{1}, r_{2}$, and $r_{3}$ are the classical turning points at energy $E . V_{0}=V_{J}\left(r_{0}\right)$ is the height of the potential maximum, and $r_{0}$ is the position of the maximum.
TABlE I. Properties of the important quasibound states of $\mathrm{H}_{2}$. Here $\nu$ is the vibrational quantum number, $J$ the rotational quantum number, $E_{i}$ the energy in $\mathrm{cm}^{-1}$ relative to the separated atoms, $\left\langle r^{2}\right\rangle_{i}^{1 / 2}$ is the root-mean-square internuclear distance in atomic units, and $\Gamma_{i}$ is the energy width of the resonance in $\mathrm{cm}^{-1}$. The $E_{i}$ and $\Gamma_{i}$ are from Ref. 29 ; the $\left\langle r^{2}\right\rangle_{i}{ }^{1 / 2}$ are from Refs. 10 and 24 .

\begin{tabular}{rrrrll}
\hline $\begin{array}{c}\text { State } \\
i\end{array}$ & $\nu_{i}$ & $J_{i}$ & $\begin{array}{c}E_{i} \\
\left(\mathrm{~cm}^{-1}\right)\end{array}$ & $\begin{array}{c}\left\langle r^{2}\right\rangle_{i}{ }^{1 / 2} \\
\text { a.u. }\end{array}$ & $\begin{array}{c}\Gamma_{i} \\
\left(\mathrm{~cm}^{-1}\right)\end{array}$ \\
\hline 1 & 14 & 5 & 44.1 & 6.55 & 17.4 \\
2 & 14 & 6 & 81.5 & 6.95 & 104. \\
3 & 13 & 8 & 86.3 & 5.4657 & 1.48 \\
4 & 13 & 9 & 191.4 & 5.95 & 52.3 \\
5 & 12 & 11 & 211.4 & 5.0189 & 2.32 \\
6 & 12 & 12 & 380.3 & 5.42 & 71.3 \\
7 & 11 & 13 & 195.5 & 4.4386 & 0.004 \\
8 & 11 & 14 & 475.7 & 4.8755 & 17.3 \\
9 & 10 & 16 & 582.0 & 4.4536 & 2.84 \\
10 & 9 & 18 & 722.4 & 4.1365 & 0.51 \\
\hline
\end{tabular}

where the (small) correction term, $\chi$, is given by

$$
\chi=\theta-\theta \ln |\theta|+\arg \Gamma\left(\frac{1}{2}+i \theta\right) \text {. }
$$

Here $\Gamma$ is the gamma function and $\theta$ is the barrier phase integral (see Fig. 1)

$$
\theta=\pi^{-1}\left(\frac{2 \mu_{\mathrm{MX}}}{\hbar^{2}}\right)^{1 / 2} \int_{\tau_{2}}^{r_{3}}\left(V_{J}-E\right)^{1 / 2} d r
$$

The energy widths, $\Gamma_{m}$, of the quasibound states were obtained from ${ }^{16}$

$\Gamma_{m}=\left[2 \pi(\partial \phi / \partial E)_{E_{m}}\right]^{-1} \ln \left\{1+\exp \left[-2 \pi \theta\left(E_{m}\right)\right]\right\}$.

The lifetime of the state, $\tau_{m}$, is related to the width by-

$$
\tau_{m}=\hbar / \Gamma_{m}
$$

For energies below the top of the barrier, the numerical integrations to determine $\phi$ and $\theta$ are well defined. Above the barrier top it can be shown ${ }^{22}$ that the upper limit of the $\phi$ integral is $r_{0}$, the position of the barrier maximum; hence, the $\phi$ integral is still easily calculated. However, the turning points $r_{2}$ and $r_{3}$ become complex ${ }^{23}$ above the barrier top, so that determination of $\theta$ by numerical integration becomes difficult. To avoid this problem, it was noted from exact solutions for simple potential barriers (parabola, inverted Morse, etc.) that $\theta$ is an analytic function of $E$ near $V_{0}=V_{J}\left(r_{0}\right)$. Hence, an analytic form was fit to $\theta(E)$ for $E<V_{0}$ and used to extrapolate to energies above but near $V_{0}$. The function,

$$
\theta=\left(V_{0} / \hbar \omega_{B}\right) \ln \left(V_{0} / E\right),
$$

where $\omega_{B}=\left(-V_{0}^{\prime \prime} / \mu_{\mathrm{MX}}\right)^{1 / 2}$ is the barrier frequency, was found to agree well with numerical values of $\theta$ calculated from realistic potentials. [For $E$ very near $V_{0}$, this reduces to the known result for a parabolic barrier, $\left.{ }^{16,23} \theta=\left(V_{0}-E\right) / \hbar \omega_{B} \cdot\right]$

Use of this approximate $\theta$ affects the calculated 
TABLE II. Parameters used in the repulsive exponential potentials of the ET calculations. Values are from Ref. 10 and are in Hartree atomic units.

\begin{tabular}{lcc}
\hline System & $\begin{array}{c}\epsilon \\
(\text { a.u. })\end{array}$ & $\begin{array}{c}a \\
\text { (a.u.) }\end{array}$ \\
\hline $\mathrm{He}-\mathrm{H}$ & 2.832 & 0.5 \\
$\mathrm{Ar}-\mathrm{H}$ & 20.93 & 0.5 \\
$\mathrm{H}_{2}-\mathrm{H}$ & 7.696 & 0.5 \\
\hline
\end{tabular}

energies of quasibound levels above the barrier negligibly since $\chi(\theta)$ in (4.5) is a small correction anyway, but its use does reduce the accuracy of the energy widths and lifetimes of levels above the barrier; they are probably good only to within a factor of 2 .

\section{CALCULATIONS AND RESULTS}

The specific reactions considered were the recombination of $\mathrm{H}$ atoms in $\mathrm{He}, \mathrm{Ar}$, and $\mathrm{H}_{2}$. The separate contributions of the $\mathrm{BC}$ and $\mathrm{ET}$ mechanisms were estimated for each of the three reactions.

\section{A. Energy-Transfer Mechanism}

The energies, $E_{i}$, and the internuclear distances, $\left\langle r^{2}\right\rangle_{i}{ }^{1 / 2}$, of the 10 contributing quasibound states of $\mathrm{H}_{2}$, originally determined by Waech and Bernstein, ${ }^{17}$ have recently been redetermined accurately by LeRoy, ${ }^{24}$ and we used his values (see Table I). The degeneracies needed in (2.5) are $g_{\mathrm{H}}=4, g_{i}=3$ if $J_{i}$ is odd, and $g_{i}=1$ if $J_{i}$ is even. The parameters in the $\mathrm{MH}$ potential (2.16) were those of RBC (see Table II). The calculations using (2.18) were easily done, and the results are plotted in Figs. 2-4 together with those of RBC. Since RBC's distorted-wave approximation is known to give an upper bound, it is seen (in Fig. 2, for example) that our

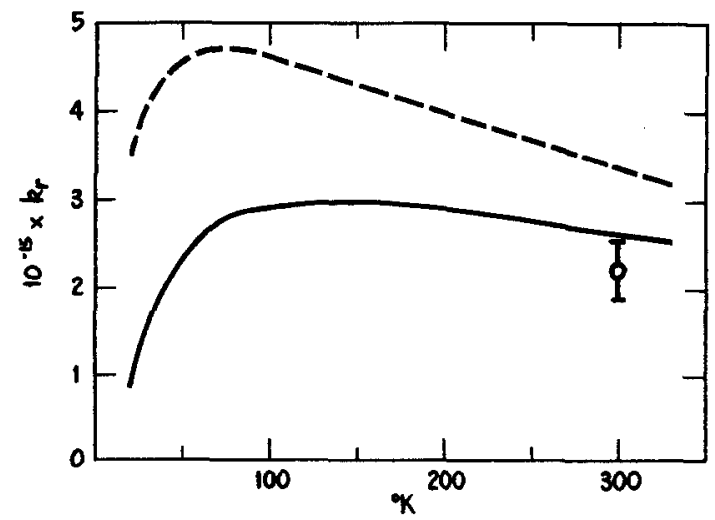

Fig. 2. Recombination rate constants for $\mathrm{H}+\mathrm{H}+\mathrm{He} \rightarrow \mathrm{H}_{2}+\mathrm{He}$ in units of $\mathrm{cm}^{6} / \mathrm{mole}^{2}$.second. In this case $k_{r}=k_{r} \mathrm{ET}$. The dashed line is the RBC results; the solid line is the estimate of the present paper. The experimental point is from J. E. Bennett and D. R. Blackmore, Symp. Combust. 13th, Salt Lake City, 1971, 51 (1971).

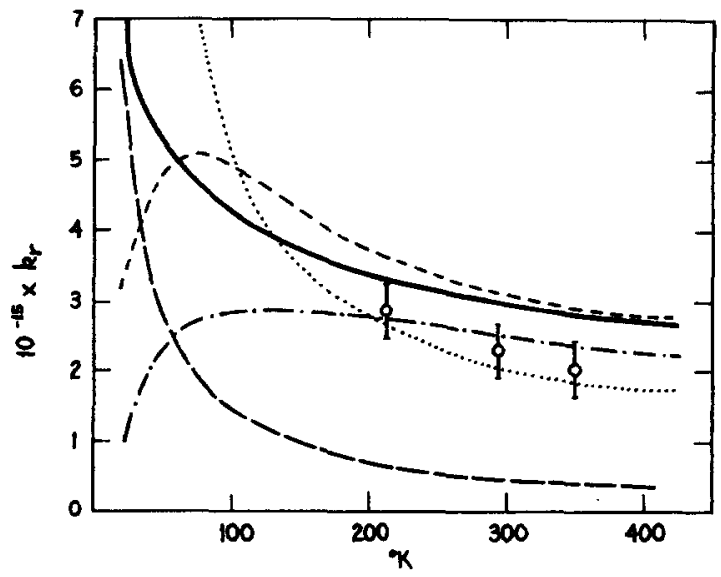

FIG. 3. Recombination rate constants for $\mathrm{H}+\mathrm{H}+\mathrm{Ar} \rightarrow \mathrm{H}_{2}+\mathrm{Ar}$ in units of $\mathrm{cm}^{6} / \mathrm{mole}^{2}$. second. Solid line, present estimate of $k_{r}=k_{r} \mathrm{BC}+k_{r} \mathbf{E T}$; long dashes, present estimate of $k_{r} \mathrm{BC}$; dot-dash, present estimate of $k_{r}{ }_{r}$ T. ; short dashes, RBC's $k_{r} \mathbf{E T}$; dotted line, results of Ref. 13; experimental points are from F. S. Larkin, Can. J. Chem. 46, 1005 (1968).

simple modifications still give reasonable estimates of $k_{r}$ ET.

\section{B. Bound-Complex Mechanism: $\mathrm{H}+\mathrm{H}+\mathrm{He} \rightarrow \mathrm{H}_{2}+\mathrm{He}$}

The best available $\mathrm{HeH}$ interatomic potential is the MC SCF potential of Das and Wahl. ${ }^{25}$ It is only $5.35^{\circ} \mathrm{K}$ deep at its equilibrium distance (6.9 a.u.) and is found to support no bound vibrational levels and no longlived quasibound rotation-vibration states. Hence, $k_{r}{ }^{\mathrm{BC}}$ calculated from (3.17) is zero at all temperatures, and the observable rate constant $k_{r}=k_{r}{ }^{\mathrm{BC}}+k_{r}{ }^{\mathrm{ET}}$ reduces to $k_{r}{ }^{\text {ET }}$ shown in Fig. 2.

\section{Bound-Complex Mechanism: $\mathrm{H}+\mathrm{H}+\mathrm{Ar} \rightarrow \mathrm{H}_{2}+\mathrm{Ar}$}

No accurate ArH potential is available. The potential used was an empirical Lennard-Jones 12-6 potential determined from the molecular-beam value ${ }^{26}$ of the strength parameter, $\epsilon r_{\epsilon}=9.60 \times 10^{-4}$ a.u., and the known

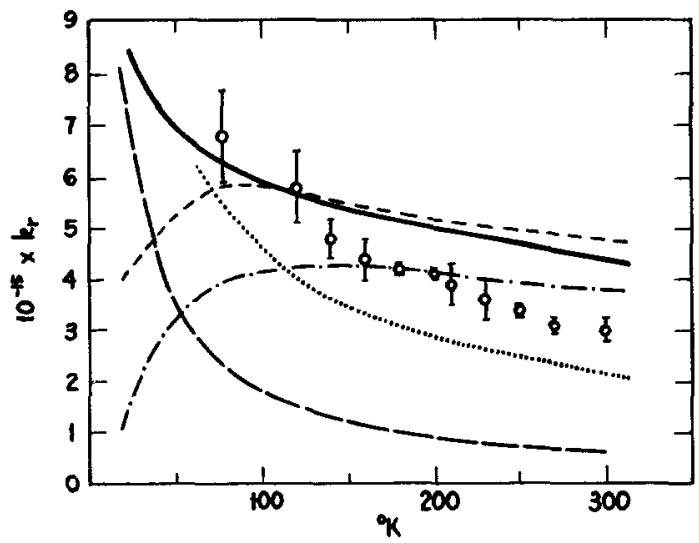

FIG. 4. Recombination rate constants for $\mathrm{H}+\mathrm{H}+\mathrm{H}_{2} \rightarrow 2 \mathrm{H}_{2}$ Units and notation are that of Fig. 3. The experimental points are from Ref. 12 . 
TABLE III. Rotation-vibration states of ArH calculated from a 12-6 potential with $\epsilon=1.510 \times 10^{-4}$ a.u. and $r_{e}=6.357$ a.u. $V_{0}$ is the height of the potential maximum in $\mathrm{cm}^{-1} ; r_{0}$ its position in atomic units. The notation on the other quantities is that of Table $I$.

\begin{tabular}{ccccccccc}
\hline \hline State $m$ & $\nu$ & $J$ & $\begin{array}{c}E_{m} \\
\left(\mathrm{~cm}^{-1}\right)\end{array}$ & $\begin{array}{c}\Gamma_{m} \\
\left(\mathrm{~cm}^{-1}\right)\end{array}$ & $\begin{array}{c}\left\langle r^{2}\right\rangle_{m}{ }^{1 / 2} \\
\text { a.u. }\end{array}$ & $\begin{array}{c}V_{0} \\
\left(\mathrm{~cm}^{-1}\right)\end{array}$ & $\begin{array}{c}\gamma_{0} \\
\text { a.u. }\end{array}$ \\
\hline 1 & 0 & 0 & -5.90 & $\ldots$ & 8.36 & 0.0 & $\ldots$. & \\
2 & 0 & 1 & -4.02 & $\ldots$ & 8.51 & 0.25 & 18.08 \\
3 & 0 & 2 & -0.467 & $\ldots$ & 8.92 & 1.30 & 13.71 \\
4 & 0 & 3 & 4.28 & 2.65 & 9.67 & 3.69 & 11.48 \\
\hline \hline
\end{tabular}

van der Waals coefficient, ${ }^{27,28} C_{6}=2 \epsilon r_{e}{ }^{6}=19.93$ a.u. These give $\epsilon=1.510 \times 10^{-4}$ a.u. $\left(47.7^{\circ} \mathrm{K}\right)$ and $r_{e}=6.357$ a.u. The ArH rotation-vibration states and their properties calculated from this potential are presented in Table III. Quasibound states whose widths were greater than half the vibrational level spacing were omitted. ${ }^{29}$

Using the data in Table III and $g_{m} / g_{\mathrm{H}} g_{\mathrm{Ar}}=1$, the $K_{\mathrm{eq}}{ }^{m}$ were calculated from (3.5). In (3.9), $r_{\mathrm{ArH}}$ was obtained from the ArH 12-6 potential and $r_{\mathrm{HH}}$ from the known $\mathrm{H}_{2}$ Morse potential. In this case $G=\frac{1}{4}$, and $b$ in (3.12) was taken to be $0.2 \AA$ as recommended by Herzfeld and Litovitz. ${ }^{30}$

The resulting $k_{r}{ }^{\mathrm{BC}}$ is plotted in Fig. 3 along with $k_{r}{ }^{\mathrm{ET}}$ and the total rate constant $k_{r}=k_{r}{ }^{\mathrm{ET}}+k_{r}{ }^{\mathrm{BC}}$. Also plotted in Fig. 3 are the available experimental values and the phase-space results of Shui and Appleton. ${ }^{13}$

\section{Bound-Complex Mechanism: $\mathrm{H}+\mathrm{H}+\mathrm{H}_{2} \rightarrow 2 \mathrm{H}_{2}$}

The model used for $\mathrm{H}_{2}-\mathrm{H}$ was very crude. As in the works of $\mathrm{RBC}^{10}$ and Shui and Appleton, ${ }^{13} \mathrm{H}_{2}$ was treated as a structureless sphere, and the $\mathrm{H}_{2}-\mathrm{H}$ interaction was taken to be a $12-6$ potential with parameters ${ }^{13}$ $\epsilon=1.203 \times 10^{-4}$ a.u. $\left(38^{\circ} \mathrm{K}\right)$ and $r_{e}=6.42$ a.u. The rotation-vibration levels of this model are listed in Table IV. The turning point $r_{\mathrm{H}_{2}-\mathrm{H}}$ was obtained from this potential; the other parameters were those used in the previous (ArH) reaction. The results are plotted in Fig. 4 along with those of Shui and Appleton ${ }^{13}$ and the experimental results of Ham, Trainor, and Kaufman. ${ }^{12}$

\section{DISCUSSION AND CONCLUSIONS}

While neither the $k_{r}{ }^{\mathrm{ET}}$ nor the $k_{r}{ }^{\mathrm{BC}}$ obtained in this work can be expected to be an accurate estimate, the two contributions were calculated in a consistent fashion, and one should be able to make a reasonable assessment of their relative importance.
The reason $k_{r}$ ET has a low-temperature maximum while $k_{r}{ }^{\mathrm{BC}}$ has none, is that the ET mechanism involves only quasibound levels $\left(E_{i} \geq 0\right)$ of $\mathrm{X}_{2}$ whose equilibrium populations become small at low temperatures, while the $\mathrm{BC}$ mechanism involves shallow but truly bound $\left(E_{m}<0\right)$ states of MX whose populations become large at low temperatures. In addition, the probability of a transition from a quasibound state $i$ to the bound region [see Eq. (2.17)] becomes small when $E_{i}$ is greater than the average collisional energy.

For the recombination of $\mathrm{H}$ atoms in $\mathrm{Ar}$ or $\mathrm{H}_{2}$, one sees from the figures that the ET mechanism dominates at high temperatures, but the $\mathrm{BC}$ mechanism dominates at low temperatures, so that no maximum in the total rate constant is predicted. Agreement with experiment is good for such a simple theory.

For the recombination of $\mathrm{H}$ in Ar we also recalculated $k_{r}{ }^{B C}$ using the two ArH potentials used by Shui and Appleton. ${ }^{13}$ Both potentials lead to a $k_{r}{ }^{\mathrm{BC}}$ as large or larger than the one shown, so that the resulting $k_{r}$ has the same behavior (no maximum) as the one shown.

For $\mathrm{H}$ recombination in $\mathrm{He}$, the Das-Wahl ${ }^{25}$ potential implies that $k_{r}{ }^{\mathrm{BC}}=0$, and we thus predict that a maximum will be observed in $k_{r}$. We note, however, that if the true $\mathrm{HeH}$ potential were as deep $\left(38.4^{\circ} \mathrm{K}\right)$ as the CI potential of Miller and Schaefer, ${ }^{31}$ which has bound states, the BC mechanism would dominate at low temperatures, and $k_{r}$ would have no maximum. To see how sensitive the existence of the maximum in $k_{r}$ is to the existence of bound $\mathrm{HeH}$ vibrational levels, $k_{\mathbf{r}}{ }^{\mathrm{BC}}$ was recalculated using a $12-6$ potential $\left(\epsilon=12.2^{\circ} \mathrm{K}\right.$ and $r_{e}=6.93$ a.u.) chosen to have only one rotationless vibrational level (bound by only $0.004^{\circ} \mathrm{K}$ ) and no long-lived quasibound levels. The resulting $k_{r}{ }^{\mathrm{BC}}$ was almost large enough to destroy the maximum in $k_{r}$, i.e., $k_{r}$ was very flat at low temperatures. Thus, we con-

TABLE IV. Rotation-vibration states of $\mathrm{H}_{2}-\mathrm{H}$ calculated from a spherical model $12-6$ potential with $\epsilon=1.203 \times 10^{-4}$ a.u. and $r_{\epsilon}=6.42$ a.u. The notation is that of Tables I and III.

\begin{tabular}{|c|c|c|c|c|c|c|c|}
\hline State $m$ & $\nu$ & $J$ & $\begin{array}{c}E_{m} \\
\left(\mathrm{~cm}^{-1}\right)\end{array}$ & $\underset{\left(\mathrm{cm}^{-1}\right)}{\Gamma_{m}}$ & $\begin{array}{c}\left\langle r^{2}\right\rangle_{m}{ }^{1 / 2} \\
\text { a.u. }\end{array}$ & $\begin{array}{c}V_{0} \\
\left(\mathrm{~cm}^{-1}\right)\end{array}$ & $\begin{array}{c}r_{0} \\
\text { a.u. }\end{array}$ \\
\hline 1 & 0 & 0 & -1.81 & $\ldots$ & 9.94 & 0.0 & $\ldots$ \\
\hline 2 & 0 & 1 & +0.09 & 0.20 & 10.91 & 0.48 & 15.78 \\
\hline 3 & 0 & 2 & 4.92 & 6.31 & 9.57 & 2.50 & 11.93 \\
\hline
\end{tabular}


clude that the maximum in $k_{r}$ is sensitive to the nature of the $\mathrm{HeH}$ potential and that experimental measurement of the rate of $\mathrm{H}$ recombination in $\mathrm{He}$ at low temperatures would give valuable information about the $\mathrm{HeH}$ potential. Observance of a clear maximum would mean that $\mathrm{HeH}$ has no bound vibrational levels. $\mathrm{Ab}$ sence of a maximum would be good evidence ${ }^{32}$ that the $\mathrm{HeH}$ potential is deep enough to support bound vibrational levels.

Shui and Appleton ${ }^{13}$ noted that the classical phasespace theory predicted no maximum in $k_{r}$ even when the $\mathrm{MH}$ potential was taken to be purely repulsive. This is probably because their classical theory gives contributions from orbiting resonances (quasibound states) of all energies whereas the true spectrum (see Table I) is highly quantized and the lowest-lying quasibound state lies $63.5^{\circ} \mathrm{K}$ above the energy of the separated atoms.

Suppose we now consider the recombination of $\mathrm{H}$ atoms in the presence of third bodies $\mathrm{M}$ other than $\mathrm{He}$, $\mathrm{Ar}$, or $\mathbf{H}_{2}$. It is expected that the $\mathrm{MH}$ potential for all such $\mathrm{M}$ (except possibly $\mathrm{Ne}$ ) will be attractive enough to have bound vibrational states. Thus, $k_{r}{ }^{\mathrm{BC}}$ should dominate at low temperatures and $k_{r}$ should have no maximum.

For the recombination of atoms $\mathrm{X}$ which are heavier than hydrogen (or deuterium ${ }^{34}$ ), the larger reduced mass results in more bound MX levels and a larger BC contribution. Furthermore, $\mathrm{X}_{2}$ has lower-lying and more closely spaced quasibound levels than $\mathrm{H}_{2}$, and the maximum in $k_{r}{ }^{\mathrm{ET}}$ occurs at much lower temperatures where the $\mathrm{BC}$ mechanism is relatively even more important. Hence, we believe it unlikely that a lowtemperature maximum will be observed in the rate of recombination of heavy atoms.

\section{ACKNOWLEDGMENTS}

We thank Professor John S. Dahler and Dr. Thomas P. Tsien for helpful suggestions. We also thank the Brigham Young University Computer Research Center for computer time.

\footnotetext{
* Research supported in part by Research Corp. and the Brigham Young University Research Fund.

1 G. E. Kimball, J. Am. Chem. Soc. 54, 2396 (1932).

2 E. Rabinowitch, Trans. Faraday Soc. 33, 283 (1937).
}

${ }^{3}$ H. S. Johnston, Gas Phase Reaction Rate Theory (Ronald, New York, 1966).

${ }^{4}$ D. L. Bunker and N. Davidson, J. Am. Chem. Soc. 80, 5090

(1958) ; D. L. Bunker, J. Chem. Phys. 32, 1001 (1960).

${ }^{5}$ G. Porter, Discussions Faraday Soc. 33, 198 (1962).

${ }^{6} \mathrm{M}$. Eusuf and K. J. Laidler, Trans. Faraday Soc. 59, 2750 (1963).

${ }^{7}$ S. K. Kim, J. Chem. Phys. 46, 123 (1967).

${ }^{8} \mathrm{H}$. Eyring, H. Gershinowitz, and C. E. Sun, J. Chem. Phys. 3, $786(1935)$.

${ }^{9}$ S. W. Benson and T. Fueno, J. Chem. Phys. 36, 1597 (1962).

${ }^{10}$ R. E. Roberts, R. B. Bernstein, and C. F. Curtiss, J. Chem.

Phys. 50, 5163 (1969); Chem. Phys. Letters 2, 366 (1968).

${ }_{11}$ F. H. Mies, J. Chem. Phys. 51, 787, 798 (1969).

12 D. O. Ham, T. W. Trainor, and F. Kaufman, J. Chem. Phys. 53, $4395(1970)$.

${ }^{13}$ V. H. Shui and J. P. Appleton, J. Chem. Phys. 55, 3126 (1971).

${ }^{14}$ V. H. Shui, J. P. Appleton and J. C. Keck, J. Chem. Phys. 53, $2547(1970)$

${ }^{15}$ V. H. Shui, J. P. Appleton, and J. C. Keck, Symp. Combust. 13th, Salt Lake City, 1971, 21 (1971).

16 J. N. L. Connor, Mol. Phys. 15, 37, 621 (1968).

${ }_{17}$ T. G. Waech and R. B. Bernstein, J. Chem. Phys. 46, 4905 (1967).

${ }^{18} \mathrm{~J}$. M. Jackson and N. F. Mott, Proc. Roy. Soc. (London) A137, 703 (1932)

19 R. T Pack and J. S. Dahler, J. Chem. Phys. 50, 2397 (1969).

${ }^{20}$ The van der Waals attraction between $\mathrm{X}$ and $\mathrm{M}_{2}, \mathrm{MX}$ and $\mathrm{M}$, or $\mathrm{X}$ and $\mathrm{MX}$ allows them to get close enough together to react before any repulsion is felt.

${ }^{21}$ Our only justification for using the same form for this factor in the BC mechanism as in the ET mechanism is that it has physically reasonable behavior. Its omission would have a negligible effect on the $k_{r}{ }^{\mathrm{BC}}$ calculated herein.

${ }_{22}^{2} \mathrm{~N}$. Fröman and P. O. Fröman, JWK B Approximation (NorthHolland, Amsterdam, 1965).

${ }^{23}$ W. H. Miller, J. Chem. Phys. 48, 1651 (1968).

${ }^{24}$ R. J. LeRoy, J. Chem. Phys. 54, 5433 (1971); University of Wisconsin Theoretical Chemistry Institute Rept. WIS-TCI387,15 January 1971.

${ }^{25}$ G. Das and A. C. Wahl, Phys. Rev. A 4, 825 (1971)

${ }^{26} \mathrm{~W}$. C. Stwalley, A. Niehaus, and D. R. Herschbach, J. Chem. Phys. 51, 2287 (1969); M. A. D. Fluendy, R. M. Martin, E. E. Muschlitz, Jr., and D. R. Herschbach, J. Chem. Phys. 46, $2172(1967)$

${ }^{27}$ R. T Pack, Chem. Phys. Letters, 5, 257 (1970).

${ }^{28}$ R. J. Bell and A. E. Kingston, Proc. Roy. Soc. (London) 88, 901 (1966).

${ }^{29}$ R. J. LeRoy and R. B. Bernstein, J. Chem. Phys. 54, 5114 (1971).

${ }^{30} \mathrm{~K}$. F. Herzfeld and T. A. Litovitz, Absorplion and Dispersion of Ulirasonic Waves (Academic, New York, 1959).

${ }_{31}$ W. H. Miller and H. F. Schaefer III, J. Chem. Phys. 53, 1421 (1970).

${ }^{32}$ It is possible that nonresonant collisions might contribute, but all estimates (see Refs. 10 and 33 ) to date have indicated that their contribution is small. It is also possible that there may be a contribution from some true three-body processes not accounted for in either of our successive two-body mechanisms.

${ }_{33} \mathrm{~J}$. L. Jackson and R. E. Wyatt, J. Chem. Phys. 54, 5271 (1971).

${ }^{34}$ R. E. Roherts and R. B. Bernstein, Chem. Phys. Letters 6, $282(1970)$ 